\title{
A Probabilistic Framework for Complex Wavelet Based Image Registration
}

\author{
Florina-Cristina Calnegru \\ University of Pitesti, Department of Computer Science \\ calnegru_florina@yahoo.com
}

\begin{abstract}
The aim of this article is to introduce a computationally tractable mathematical model of the relation between the complex wavelet coefficients of two different images of the same scene. Because the two images are acquisitioned at distinct times, from distinct viewpoints, or by distinct sensors, the relation between the wavelet coefficients is far too complex to handle it in a deterministic fashion. This is why we consider adequate and present a probabilistic model for this relation. We further integrate this probabilistic framework in the construction of a new image registration algorithm. This algorithm has subpixel accuracy, and is robust to noise and to a large class of local variations like changes in illumination and even occlusions. We empirically prove the properties of this algorithm using synthetic and real data.
\end{abstract}

Keywords: Image registration, probabilistic similarity measure, complex wavelet transform.

\section{Introduction}

In Visual Computing, next to the problem of analyzing a single image, one often encounters the problem of combining information contained in more images [1]. The first step in integrating the information comprised in a set of images is the registration of each pair of images from that set.

Image registration is the process of geometrically overlapping two images of the same scene, obtained at different moments in time, or from different view angles, or with different sensors [2]. The two images involved in the process of registration are the reference image and the target image or the sensed image. With this terminology, the registration can be defined as the process of finding a transformation such that the target image becomes similar with the reference image [3].

Largely speaking there are two kinds of registration methods: parametric and nonparametric. In the case of the parametric registration, the transformation is parametric, i.e. can be expanded in terms of some basis functions. In the case of non-parametric registration, the transformation is no longer restricted to a parametrizable set. As the algorithm that we propose is from the category of parametric image registration, we will no further insist on the non-parametric image registration. Parametric image registration can be divided into: landmark based parametric image registration, principal axes-based registration, and optimal parametric registration [1].

Landmark based parametric registration is a type of registration based on the features extracted at an initial stage in the process of registration. Those features can be 
lines intersections, road crossings, inflection points of curves, corners [2], local extremes of wavelet transform [4], etc. The quality of landmark-based registration is highly dependent on the performances of the feature detector that is used. If the feature detector is not reliable enough, the registration will be low quality.

This is why sometimes is better to use a registration method that relays on features that can be automatically deduced from the image. Such intrinsic features are for example the principal axes [5]. Although principal axes registration is fast and necessitates very few parameters, it needs the moment matrix and the eigenvalue decomposition of two large matrixes, it is not suitable for multimodal registration, and its results can be ambiguous [1].

The disadvantages of the landmark-based registration and principal axes registration led to the emergence of a more general and flexible class of parametric image registration. This category consists of optimal parametric registration algorithms.

The basic idea of optimal parametric registration is to define a distance (similarity) measure between the reference image and the target image, and then to find the parameters of the transformation that optimize this similarity measure. The most known similarity measures are the sum of squared differences, the correlation, and the mutual information [6]. The domain of these similarity measures is either the intensity space like in [7], or another feature space like the wavelet coefficients with a magnitude above a certain threshold [4], the energy map [8], and the wavelet coefficients from the first decomposition level [9].

The algorithm that we introduce is an optimal registration algorithm. The similarity measure that we use is defined on the complex wavelet coefficients space. This makes our similarity measure more robust to noise than the similarity measures defined on the intensity space. The later mentioned similarity functions are affected by the noise, that usually corrupts the image intensities.

This article is structured as following. In section 2 we present an introduction to the dual-tree complex wavelet transform. In section 3 we expose the probabilistic model underlying the registration algorithm. In section 4 we present the registration algorithm and introduce different modalities to integrate it into real world registration systems. In section 5 we justify the necessity for our mathematical model in the present context, which is that of the existence of a related well-known probabilistic model in the intensity domain. In section 6 we present experimental results on artificial data as well as on real data. In section 7 we present our conclusions.

\section{Complex Wavelet Transform}

Discrete wavelet transform (DWT) is a modality to project a signal onto an orthogonal wavelet basis. By using the DWT one can obtain local information about a signal both in the spatial domain and in the frequency domain. For a 2-D signal the DWT coefficients are obtained by passing the signal through a cascade of orthogonal high pass and low pass filters. The original image is decomposed at any scale $\mathrm{j}$, into 4 components: $\mathrm{HH}_{\mathrm{j}}$ (contains the diagonal details), $\mathrm{HL}_{\mathrm{j}}$ (contains the horizontal details), $\mathrm{LH}_{\mathrm{j}}$ (comprised of vertical details), and $\mathrm{LL}_{\mathrm{j}}$ (contains the approximation coefficients). For more information on DWT see [10]. 
Any signal $f(x, y)$ can be reconstructed via the inverse discrete wavelet transform from its detail and approximation coefficients as in (1)

$$
\begin{aligned}
f(x, y) & =\frac{1}{\sqrt{M N}} \sum_{m} \sum_{n} W_{\varphi}\left(j_{0}, m, n\right) \varphi_{j_{0}, m, n}(x, y) \\
& +\frac{1}{\sqrt{M N}} \sum_{k=1}^{3} \sum_{j=j_{0}}^{\infty} \sum_{m} \sum_{n} W_{\psi}^{k}(j, m, n) \psi_{j, m, n}^{k}(x, y)
\end{aligned}
$$

In (1) $\varphi_{\mathrm{j}, \mathrm{m}, \mathrm{n}}$ represents the scaling function scaled with a factor of $\mathrm{j}$ and translated with $\mathrm{m}$ and $\mathrm{n}, \psi_{\mathrm{j}, \mathrm{m}, \mathrm{n}}^{\mathrm{k}}$ represents $\mathrm{k}$-th mother wavelet function, scaled with a factor of $\mathrm{j}$ and translated with $\mathrm{m}$ on $\mathrm{Ox}$, and $\mathrm{n}$ on $\mathrm{Oy}, \mathrm{W}_{\varphi}(.$, .) represents the aproximation coefficients, and $\mathrm{W}_{\psi}{ }^{\mathrm{k}}(.$, . .) represents the detail coefficients.

For the 2-D DWT there are 3 mother wavelet functions: one that permits the extraction of horizontal details, one for the vertical details, and one for the diagonal details. So we can say that $\mathrm{W}_{\varphi}(\mathrm{j}, .,$.$) corresponds to \mathrm{LL}_{\mathrm{j}}(.,$.$) and that, for example,$ $\mathrm{W}_{\psi}{ }^{1}(\mathrm{j}, .,$.$) corresponds to \mathrm{HL}_{\mathrm{j}}(.,),. \mathrm{W}_{\psi}{ }^{2}\left(\mathrm{j}, . .\right.$, .) corresponds to $\mathrm{LH}_{\mathrm{j}}(.,$.$) , and that$ $\mathrm{W}_{\psi}^{3}(\mathrm{j}, .,$.$) corresponds to \mathrm{HH}_{\mathrm{j}}(.,$.$) .$

Unfortunately, the DWT has some major drawbacks that make it less appropriate for registration. Among those drawbacks, we mention poor directional selectivity, as the $\mathrm{HH}$ coefficients cannot differentiate between edges at 45 degrees and edges at 135 degrees and rotation and translation variance. Complex wavelet transform constitutes a remedy for these problems.

One can observe that by taking in (1), instead of a real scaling function and real wavelet functions, a complex scaling function, and complex wavelet functions, for which the real and the imaginary part form a Hilbert pair, the drawbacks of the DWT are eliminated [11].

In our article, we employed dual tree complex wavelet transform to obtain the complex wavelet decomposition for our images. The dual tree complex wavelet transform uses 6 complex mother wavelets that distinguish spectral features oriented at $\left\{75^{\circ}, 45^{\circ}, 15^{\circ},-75^{\circ},-45^{\circ},-15^{\circ}\right\}$. By projecting the image onto the 6 complex wavelet functions, we obtain 6 complex wavelet coefficients for each scale and translation.

To facilitate the presentation, from now on, every time we mention wavelet transform, we refer to the dual tree complex wavelet transform.

\section{Construction of the Probabilistic Framework}

The research underlying this article is driven by the desire to understand the relation between corresponding complex wavelet coefficients of two images of the same scene. What happens with the complex wavelet coefficients when the two images are captured at different times, from different viewpoints, or with different sensors? In the following, we propose a mathematical model for this relation.

The intuition behind this model is that for each level of wavelet decomposition, the layers of magnitudes of the coefficients from one image, should have the same configuration as the layers of magnitudes of the coefficients from the other image. (For 
every image, each level of decomposition has 6 layers of coefficient magnitudes, one for each of the 6 mother wavelets.) This means that if a layer, from one image, has a certain area with large (or small) coefficient magnitudes, the corresponding layer from the other image has the same area (i.e. the area located at identical coordinates) populated with large (or small) coefficient magnitudes. More than that, we expect this to happen even at the finest granularity level, i.e. we expect that large (or small) coefficient magnitudes from one image correspond to large (or small) coefficient magnitudes from the other image. We base our expectations on the fact that wavelet coefficients are "the wavelet's response" to the structures from the image. If two images depict the same scene, then the wavelet response should be similar. This observation is sustained by a known property of this type of wavelet transform. That property says that the coefficient magnitudes should be large for wavelets that overlap singularities and should be small on smooth regions [11].

Let us denote by $M_{j, k, \psi}(m, n)$ the magnitude of the coefficient $W_{\psi}{ }^{k}(j, m, n)$. Then, by denoting $\mathrm{M}_{\mathrm{j}, \mathrm{k}, \psi}^{1}$, and $\mathrm{M}_{\mathrm{j}, \mathrm{k}, \psi}^{2}$. the coefficient magnitudes for the two images, we propose the following mathematical model for their relation:

$$
M_{j, k, \psi}^{2}(m, n)=M_{j, k, \psi}^{1}(m, n)+N\left(0, \sigma_{j, k}\right), \forall k, j, m, n
$$

$N\left(0, \sigma_{j, k}\right)$ is a Gaussian random variable of mean 0 and variance $\sigma_{\mathrm{j}, \mathrm{k}}$, and can be viewed as an admissible difference between 2 magnitudes of coefficients, that contain information about the same scene structures. Those differences can be thought to be organized in layers and levels, in the same way in which the associated magnitudes are organized. We use this model to define a probabilistic similarity measure between two images of the same scene, that differ one from another by a parametric coordinate transformation, described by the set of parameters $\theta$. From now on, we mention that transformation as $\mathrm{T} \theta$. We define the probability that a coefficient from image 1 contains information about the same scene structures as a coefficient from image 2, when image 2 is transformed with $\mathrm{T} \theta$ :

$$
p_{j, k, m, n}^{\theta}=\frac{1}{\sqrt{2 \pi \sigma_{j, k}}} e^{-\frac{1}{2}\left(\frac{M_{j, k, \psi}^{2, \theta}(m, n)-M_{j, k, \psi}^{1}(m, n)}{\sigma_{j, k}}\right)^{2}}, \forall k, j, m, n
$$

We assume that the differences, between the magnitudes of 2 correspondent layers, (i.e. of any layer of differences) are independent. We define the probability that one layer of coefficients, from one image, has the same configuration as the correspondent layer of coefficients from the other image, when this image is transformed by $\mathrm{T} \theta$ :

$$
p\left(M_{j, k, \psi}^{2, \theta} \approx M_{j, k, \psi}^{1}\right)=\prod_{m, n} p_{j, k, m, n}^{\theta}, \forall j, k
$$

We also assume that every 2 layers of differences from a level and every 2 levels of differences are independent. We obtain the probability that the coefficient magnitude of image 1 represent the same structures of the scene as the coefficients of the image 2 , when image 2 is transformed by $\mathrm{T} \theta$ : 


$$
p\left(M_{\psi}^{2, \theta} \approx M_{\psi}^{1}\right)=\prod_{j, k} p\left(M_{j, k, \psi}^{2, \theta} \approx M_{j, k, \psi}^{1}\right)
$$

Since any image can be reconstructed from its wavelet coefficients, (5) can be thought as the probability that image 1 is similar with image 2 , when image 2 is transformed with $\mathrm{T} \theta$.

It can be shown as in [12] that the maximization of (4), $\forall j, k$ is equivalent to the maximization of the cross-correlation (6) between the coefficients of the layer $\mathrm{k}$ from level $\mathrm{j}, \forall j, k$.

$$
C C_{j, k}^{\theta}=\frac{\sum_{m=1}^{M} \sum_{n=1}^{N}\left(M_{j, k, \psi}^{2, \theta}(m, n)-\overline{M_{j, k, \psi}^{2, \theta}}\right)\left(M_{j, k, \psi}^{1}(m, n)-\overline{M_{j, k, \psi}^{1}}\right)}{\sqrt{\sum_{m=1}^{M} \sum_{n=1}^{N}\left(M_{j, k, \psi}^{2, \theta}(m, n)-\overline{M_{j, k, \psi}^{2, \theta}}\right)^{2} \sum_{m=1}^{M} \sum_{n=1}^{N}\left(M_{j, k, \psi}^{1}(m, n)-\overline{M_{j, k, \psi}^{1}}\right)}},
$$

where $\overline{M_{j, k, \psi}^{2, \theta}}$ is the mean of the layer $M_{j, k, \psi}^{2, \theta}$ and $\overline{M_{j, k, \psi}^{1}}$ is the mean of the layer $M_{j, k, \psi}^{1}$.

More, it can be shown that the probability, that the 2 layers are equivalent, increases with the increase of $C C_{j, k}^{\theta}$. This means that $C C_{j, k}^{\theta}$ can be considered an indicator for the probability of equivalence between the 2 layers. Because of that and because $C C_{j, k}^{\theta}$ can be negative, we make the following approximation, and define the similarity between image 1 and image 2 , when image 2 is transformed by $\mathrm{T} \theta$ :

$$
p^{\theta}=\prod_{j, k} d_{j, k}, \text { where } \mathrm{d}_{j, k}= \begin{cases}\frac{1}{1-C C_{j, k}^{\theta}}, & \text { if } C C_{j, k}^{\theta} \neq 1 \\ c \quad, \text { otherwise }\end{cases}
$$

In the definition for $\mathrm{d}_{\mathrm{j}, \mathrm{k}}$ from (7), $\mathrm{c}$ is a large real number.

We name the similarity measure from (7) wavelet layers correlation.

\section{Registration Algorithm}

The registration algorithm consists in finding $\theta=\left(\theta_{1}, \ldots, \theta_{n}\right)_{n>0}$ such that $\mathrm{p}^{\theta}$ is maximized. To avoid that the algorithm outputs a local maximum instead of a global maximum, we used simulated annealing [7]. 
We have used this algorithm to register images that differ by an affine transform or by a similarity transform ( $\mathrm{n}$ is 6 respectively 4 ). For these images, the search space of the parameters is quite large. Fortunately, $\mathrm{p}^{\theta}$ allows us to find the optimizing parameters, not by searching on the Cartesian product of the spaces of all parameters, but by searching, in turn, on the space of each parameter. This process necessitates several iterations, depending how far the solution is from the initial guess.

We have empirically found that it is sometimes better not to use, in (7), all the levels of decomposition. Actually the number of levels it is dependent on the type (for example retinal or outdoor) of images to register.

For high temperature the generation function, for our variant of the simulated annealing algorithm, is the product between a Gaussian centered in the current value, $\mathrm{x}$, and the function $h$ from (8). For smaller temperatures, the generation function is simply a Gaussian centered in $\mathrm{x}$.

$$
h(x)=\left\{\begin{array}{l}
1, \mathrm{r}<0.5 \\
-1, \mathrm{r} \geq 0.5
\end{array}, \text { where } \mathrm{r} \in(0,1)\right. \text { is a uniform random number }
$$

The speed of our algorithm depends on three factors. The first is the initial value for the set of parameters $\theta$. The second is the stopping criterion. We have used as the stopping criterion the value of $\mathrm{p}^{\theta}$ as long as a number of iterations is not reached. The third speed factor is the number of parameters from the set $\theta$. If this number is large, then the algorithm is more time costly.

Our algorithm can be used in many situations:

1. One can use it to estimate the vector $\theta$, starting from a random value for this vector. When the vector $\theta$ has a single parameter, for example the rotation angle, the speed is reasonable and the algorithm can be considered even for time dependent applications. If the registration task consists in finding a transformation with a large number of parameters, we recommend our algorithm when the time is not crucial, but instead the registration accuracy is. This recommendation is supported by the fact that our algorithm permits the finding of a solution with high accuracy.

2. One can use the algorithm for tuning the solution vector $\theta$. This assumes of course that a less accurate solution was already found by a different method. For example as in [13], one can find a first alignment from low frequency components. In this situation, the speed of convergence to a highly accurate solution is reasonable for time dependent applications even when the number of parameters is large.

\section{Wavelet Layers Correlation versus Classical Cross-Correlation}

We have arrived at wavelet layers correlation by imposing the condition (2) on corresponding wavelet coefficients. If one imposes a similar condition on the intensity values of corresponding pixels, then one obtains as a similarity measure the normalized cross-correlation. The question is why should anyone impose a condition of type (2) on wavelets coefficients rather than on intensities? Therefore, why should anyone use 
wavelet layers correlation rather than use the classical cross-correlation? We will endeavor to answer this question in the current section.

There are two major reasons for which wavelet layers correlation is superior to classical correlation:

1. A relation of type (2) is, in general, non-realistic for image intensities, as most of the times the noise is non-Gaussian. In exchange, because we imposed a different relation of type (2) for every layer of wavelet coefficients, our model can tackle a more general and realistic type of noise than the Gaussian noise. This phenomenon is more obvious in the case of multimodal images. Fig. 2 shows cross-correlation and wavelet layer correlation as functions of the shift on Ox-axis, for a multimodal retina pair of images. One can see in Fig. 2 that wavelet layers correlation attains the maximum around the correct value, which is 1.03 , while cross correlation loses the right solution completely.
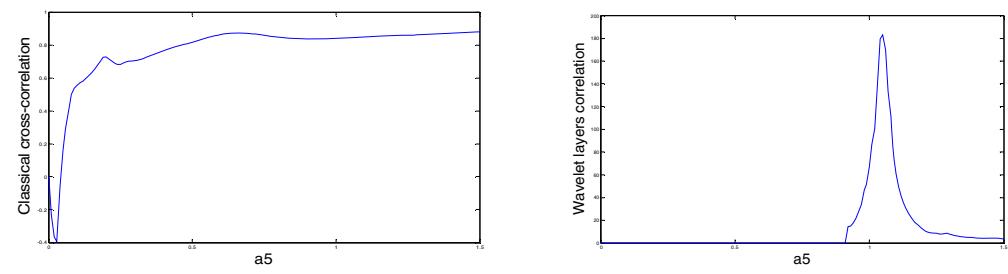

Fig. 1. The cross correlation (left) and the wavelet layers correlation (right) as functions of the 5-th parameter of the set $\theta=(\mathrm{a} 1, \mathrm{a} 2, \mathrm{a} 3, \mathrm{a} 4, \mathrm{a} 5, \mathrm{a} 6) . \theta$ describes the affine transform that registers a pair of multimodal retina images. The values of the other five parameters are fixed to their correct values. These correct values are computed from the ground truth.

2. Even when a model of type (2) can be applied to intensities, cross-correlation is a flatter similarity function than the wavelet layers correlation. This means that the classical correlation has more local maxima than the wavelet layers correlation. It also means that in the case of classical cross-correlation, the global maximum is less conspicuous than in the case of wavelet layers correlation (see Fig. 3). This increases, for an optimization method, the risk of being caught in a local maximum.
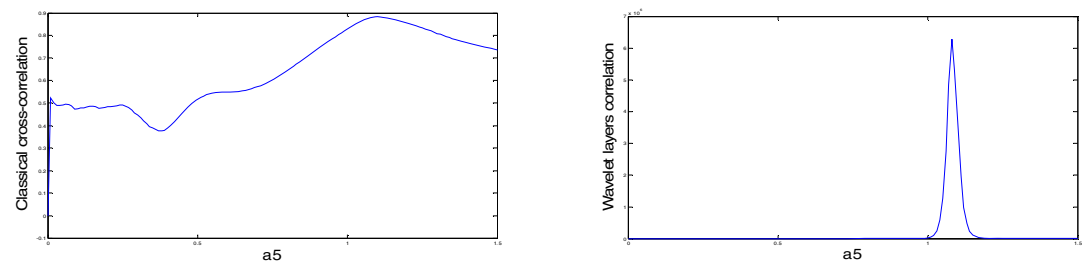

Fig. 2. The cross correlation (left) and the wavelet layers correlation (right) as functions of the 5-th parameter of the set $\theta=(\mathrm{a} 1, \mathrm{a} 2, \mathrm{a} 3, \mathrm{a} 4, \mathrm{a}, \mathrm{a})$ ). $\theta$ describes the affine transform that registers a pair of single modality retina images. The values of the other five parameters are fixed to their correct values. These correct values are computed from the ground truth. 


\section{Experimental Results}

\subsection{Synthetic Data}

In order to test the algorithm on synthetic data we have performed two kinds of tests:

1. We took some images and transformed them randomly by a single parameter transform (i.e. either by a rotation, with a random angle between $-90^{\circ}$ and $90^{\circ}$, either by a random translation between 0 pixels and half the image size, and either by a scaling with a random scaling factor between 0.1 and 3). For every pair of images we chose randomly the type of the transform. We have produced 100 pairs of images. Fig. 3 (left) shows an example of such a pair. The inaccuracy for this pair, as defined in (9), is 0.21 pixels.
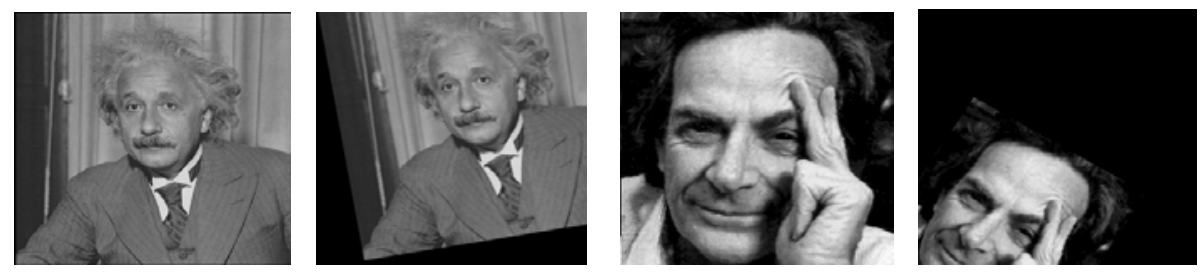

Fig. 3. Example of artificial image pairs. The second image from the left pair is the first image from the left, rotated with $-9.84^{\circ}$. The first image from the right side is the second image from the right, transformed by a similarity transform.

2. We took the same images and transformed them randomly by a similarity transform. This consists in scaling, together with translation and rotation. The scaling factor is between 0.2 and 2 , the rotation angle is between $-75^{\circ}$ and $75^{\circ}$, and the translation is between 0 and half the image size. We obtained 50 pairs. You can see in Fig. 3 (right) an example of such a pair. The inaccuracy for this pair, computed accordingly to (9), is 0.32 pixels.

If we denote, for a pair of images, $\left(\mathrm{I}_{1}, \mathrm{I}_{2}\right)$, by $\mathrm{T} \theta_{\mathrm{t}}$ the real transform (the transform that is used to obtain $\mathrm{I}_{2}$ from $\mathrm{I}_{1}$ ) and by $\mathrm{T} \theta_{\mathrm{a}}$ the transform outputted by the algorithm, then the inaccuracy for that pair is given in (9):

$$
\sum_{i=1}^{P} \frac{\left\|T \theta_{t}\left(x_{i}, y_{i}\right)-T \theta_{a}\left(x_{i}, y_{i}\right)\right\|_{2}}{P},\left(x_{i}, y_{i}\right)_{i=\overline{1, P}} \text { are random points in } \mathrm{I}_{1}
$$

Table 1. The results of the tests on synthetic data

\begin{tabular}{|l|c|}
\hline $\begin{array}{l}\text { Type of the } \\
\text { artificial transform }\end{array}$ & $\begin{array}{l}\text { Percent of image pairs registered by wavelet } \\
\text { layers correlation with an inaccuracy < } \\
\text { pixel }\end{array}$ \\
\hline Single parameter & $100 \%$ \\
\hline Multiple parameter & $98 \%$ \\
\hline
\end{tabular}




\subsection{Real Data}

We have used three categories of real world images: single modality retina images, multimodal retina images, and outdoor images. The retinal images are considered to differ by an affine transform and the outdoor images by a similarity transform.

The left side of Fig. 4 represents an example of outdoor pair on which we tested the algorithm. The right side of Fig. 4 shows the overlap of the two images from the left side, before registration, and after registration, by means of our registration algorithm. From Fig. 4 we can see that the algorithm is robust to small occlusions, as the man in front of the exit door from the second image, from the left side, is absent in the first image. This visual satisfactory behavior of the algorithm is supported by the accuracy test, which outputs for this pair an inaccuracy of 3.96 pixels.
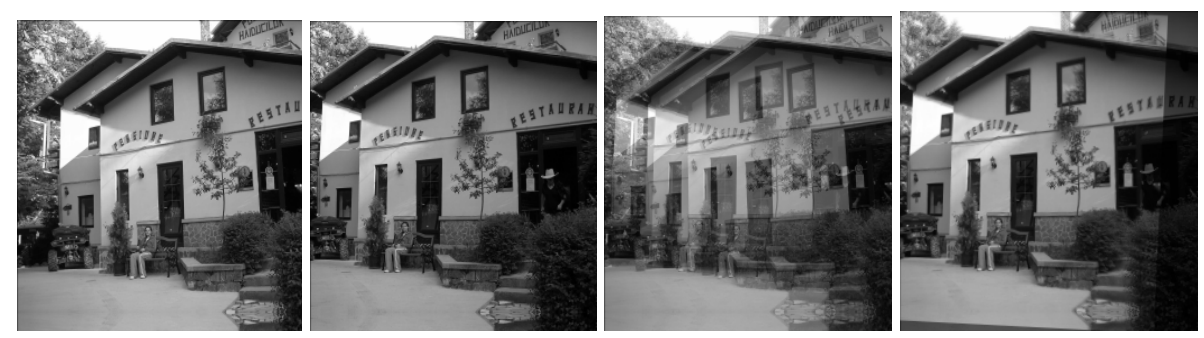

Fig. 4. The first two images from the left side represent an example of outdoor pair of images that we employed to test the algorithm. The second image from the right side represents the two images, from the left side, overlapped without registration. The first image from the right side represents the two images, from the left side, overlapped after registration with our algorithm.

In order to test the accuracy on a pair of real images, we employed the formula (9) in which $\mathrm{T} \theta_{\mathrm{t}}$ (the true transform) is computed from the ground truth (i.e. some correspondent manual chosen points in every pair of images). We used in our tests 2000 random points (i.e. $\mathrm{P}=2000$ in formula (9)) in order to probe the accuracy.

We tested the algorithm on 50 outdoor image pairs, on 50 single modality image pairs, and on 30 multimodal image pairs. The outdoor images were captured with an Olympus Camedia C-500 Zoom camera. The retina images were provided by OD-OS $\mathrm{GmbH}$.

We implemented the algorithm in MATLAB on a Intel(R) Core (TM) 2 CPU T5300, $1.73 \mathrm{GHz}$ processor. We obtained the results summarized in Table 2.

Table 2. Results of the tests on real data

\begin{tabular}{|l|c|}
\hline Image cathegory & $\begin{array}{l}\text { Percent of image pairs registered by } \\
\text { wavelet layers correlation with an } \\
\text { inaccuracy < pixels }\end{array}$ \\
\hline Oudoor & $98 \%$ \\
\hline Single modality retinal & $94 \%$ \\
\hline Multimodal retinal & $93.3 \%$ \\
\hline
\end{tabular}




\section{Conclusions}

In this paper we have proposed a probabilistic framework for modeling the relation between correspondent wavelet coefficients. We have used this framework for creating a new registration algorithm. The main qualities of this algorithm are: large applicability (can be employed for a variety of images ( as section 6 shows), and in many situations (described in section 4), robustness to small occlusions (as the tests on outdoor images show), robustness to a large category of noise (as can be seen from the tests on single modality and multiple modality image), the ellimination of any neccesity for preprocessing like for example noise reduction (this can be seen from all our tests since we have used no preprocessing), and sub-pixel accuracy (this was proved only on synthetic images since the accuracy on real images (as is outputed by the tests) depends on the limited precision of the human individuals that created the ground truth).

\section{References}

1. Modersitzki, J.: Numerical Methods for Image Registration. Oxford University Press, New York (2004)

2. Zitova, B., Flusser, J.: Image registration methods: a survey. Image and Vision Computing 21(11), 977-1000 (2003)

3. Goshtasby, A.: 2-D and 3-D Image Registration for medical, remote sensing, and industrial applications. John Wiley and Sons, Inc., Hoboken (2005)

4. Moigne, J.L., Campbell, W.J., Cromp, R.F.: An automated parallel image registration technique based on the correlation of wavelet features. IEEE Trans. On Geoscience and Remote Sensing 40(8), 1849-1864 (2002)

5. Alpert, N.M., Bradshaw, J.F., Kennedy, D., Correia, J.A.: The principal axes transformation A method for image registration. Journal of Nuclear Medicine 31(10), 1717-1722 (1990)

6. Viola, P., Wells III, W.: M.: Alignment by maximization of mutual information. In: International Conference on Computer Vision, pp. 16-23 (1995)

7. Ritter, N., Owens, R., Cooper, J., Eikelboom, R.H., van Saarloos, P.: Registration of Stereo and Temporal Images of the Retina. IEEE Transactions on Medical Imaging 18(5) (1999)

8. Pauly, O., Padoy, N., Poppert, H., Esposito, L., Navab, N.: Wavelet energy map: A robust support for multi-modal registration of medical images. In: IEEE Conference on Computer Vision and Pattern Recognition, pp. 2184-2191 (2009)

9. Li, S., Peng, J., Kwok, J.T., Zhang, J.: Multimodal registration using the discrete wavelet frame transform. In: Proc. of ICPR Conf., pp. 877-880 (2006)

10. Gonzalez, R., Richard, R.: Digital Image Processing. Prentice Hall, Upper Saddle River (2002)

11. Selesnick, I.W., Barniuk, R.G., Kingsbury, N.G.: The Dual-Tree Complex Wavelet Transform. IEEE Signal Processing Magazine (2005)

12. Nixon, M.S., Aguado, A.S.: Feature Extraction and Image Processing. ButterworthHeinemann/Newnes, Oxford (2002)

13. Indian Institute of Information Technology, Allahabad,

http://mtech.iita.ac.in/Agrade/

Sukriti-MedicalImageRegistrationusingNextGenerationWavelets.pdf 